CASE REPORT

\author{
Y.-K. Ku \\ H.-Wu Chen \\ H.-Wen Chen \\ C.-J. Fu \\ S.-C. Chin \\ Y.-C. Liu
}

\section{Giant Extracranial Aneurysms of Both Internal Carotid Arteries with Aberrant Jugular Veins in a Patient with Neurofibromatosis Type 1}

\begin{abstract}
SUMmARY: Although neurofibromatosis type 1 (NF-1) is commonly considered neurocutaneous, severe arterial and venous abnormalities have been noted. Our patient, a 28-year-old woman, had bilateral giant extracranial aneurysms of the internal carotid arteries as well as skull base meningoceles involving the jugular foramina and aberrant jugular veins. CT and MR imaging, as well as digital subtraction and/or other angiography techniques, may be required to clarify pathology in patients with suggested vascular lesions.
\end{abstract}

T he autosomal dominant mutation underlying neurofibromatosis type 1 (NF-1) has a very high prevalence, affecting 1 in 3000 Americans. ${ }^{1,2}$ Although NF-1 is defined by neurocutaneous findings, severe abnormalities of bone, ${ }^{1}$ arteries, ${ }^{2,3}$ and veins ${ }^{4}$ have been noted. We describe a 28 -year-old patient with bilateral giant extracranial aneurysms of the internal carotid arteries (ICAs), skull base meningoceles involving the jugular foramina, and aberrant jugular veins, who was treated successfully: After obtaining detailed imaging studies, we sacrificed her left ICA and used intravascular trapping with $\mathrm{Mi}$ croCoils (MicroCoil System; Micrus, San Jose, Calif) in the right ICA and an endoluminal stent graft in the right external carotid artery (ECA) to bridge the orifice of the right ICA and exclude internal carotid flow. We discuss the pathogenesis of this multisystem disease to emphasize the importance of using MR imaging and CT techniques to clarify the lesions present in patients with suggested vascular abnormalities.

\section{Case Report}

A 28-year-old woman with skin hyperpigmentation, multiple softtissue masses bilaterally at the neck, and multiple café-au-lait spots on the trunk since 3 years of age was diagnosed with NF-1. Her bilateral neck plexiform neurofibromas had grown slowly and were accompanied by cervical neuralgia. Progressive dysarthria and dysphagia and minimal right hemiparesis had also developed. Two years previously, the patient had experienced a large left middle cerebral artery (MCA) infarction.

At admission, 2 large masses with bruit were noted bilaterally at the upper neck. Neurologic examination revealed dysfunction of cranial nerves IX-XII, with symptoms including dysphagia, dysarthria, hoarseness, and weakness of the left trapezius muscle. In addition, tongue deviation to the left on protrusion and right lower extremity spasticity were noted; these were considered sequelae of the infarction.

MR imaging confirmed the large left MCA infarct near the left

Received December 9, 2007; accepted after revision March 17, 2008

From the Department of Medical Imaging and Intervention (Y.-K.K., H.-Wu C., C.-J.F., S.-C.C., Y.-C.L.), Chang Gung Memorial Hospital, Linkou, Taiwan; Department of Medical Imaging and Radiological Sciences (Y.-C.L.), Chang Gung University, Taiwan; and Department of Internal Medicine (H.-Wen C.), Lo-Tung Poh-Ai Hospital, Yilan, Taiwan.

Please address correspondence to Yuan-Chang Liu, MD, Department of Medical Imaging and Intervention, Chang Gung Memorial Hospital, Department of Medical Imaging and Radiological Sciences, Chang Gung University, 5 Fu-Hsing St, Gueishan, TaoYuan, 33305, Taiwan; e-mail: b2401003@gmail.com

DOI 10.3174/ajnr.A1135 lateral ventricle and revealed recent infarcts in the left cerebral hemisphere, a giant aneurysm of the left cervical ICA $(4 \times 5 \mathrm{~cm}$ in the transverse plane, $8 \mathrm{~cm}$ long) with mural thrombus, and another infarct of the right cervical ICA $(3 \times 4 \mathrm{~cm}$ in the transverse plane, $5 \mathrm{~cm}$ long) (Fig 1), as well as infiltrative plexiform neurofibromas in the neck extending between the muscles of the left shoulder. A short stenotic segment of the right extracranial ICA was observed above the aneurysm tip. CT angiography was performed when the patient developed tachycardia and sudden-onset left shoulder pain and swelling, suggesting possible aneurysmal rupture. A large hematoma was noted in the soft tissue of the left shoulder, but there was no extravasation of contrast. Additionally, CT indicated 5 skull defects: It revealed bilateral defects at the lambdoid sutures and jugular foramina, with hypoglossal canals, and at the occipital protuberance (Fig 2), and bilateral aneurysmal dilations of the aberrant jugular veins from the sigmoid sinus passing through the lambdoid sutures (Fig 3).

Conventional angiography showed good collateral flow intracranially and bilaterally from the ECAs and minimal flow through the ICAs. We sacrificed her left ICA to prevent aneurysmal growth and stroke due to thrombotic embolism. We decided to perform intravascular trapping of the right ICA and aneurysm by using MicroCoils and placement of a graft stent in the right ECA, bridging the orifice of the right ICA to exclude internal carotid flow. The procedure was uneventful, and the patient was discharged without symptoms beyond those present at admission.

\section{Discussion}

Despite the common association of NF-1 with neurocutaneous features, its pathology can extend to numerous tissues not derived from the neural crest. In our literature search, we identified articles focusing on vasculopathy as a common, potentially serious, and generally under-recognized component of NF-1. ${ }^{4-6}$ Recent research on the morbidity and mortality in NF-1 disclosed 2 factors independently associated with an excess of mortality in children and adults under 30 years of age: soft-tissue malignancies and vascular disease. ${ }^{5}$ Our patient was 28 years old. Case reports on vascular anomalies have described severe intracranial and extracranial abnormalities involving arteries and occasionally, veins, often in combination and life-threatening in nature. .,3,7-10 $^{-1}$

Sometimes vascular lesions are clearly related to neurofibromas. Either vessels within the neurofibroma are anomalous or a neurofibroma compresses or infiltrates adjacent blood vessels. ${ }^{2,6}$ The aneurysms in our patient's neck were 

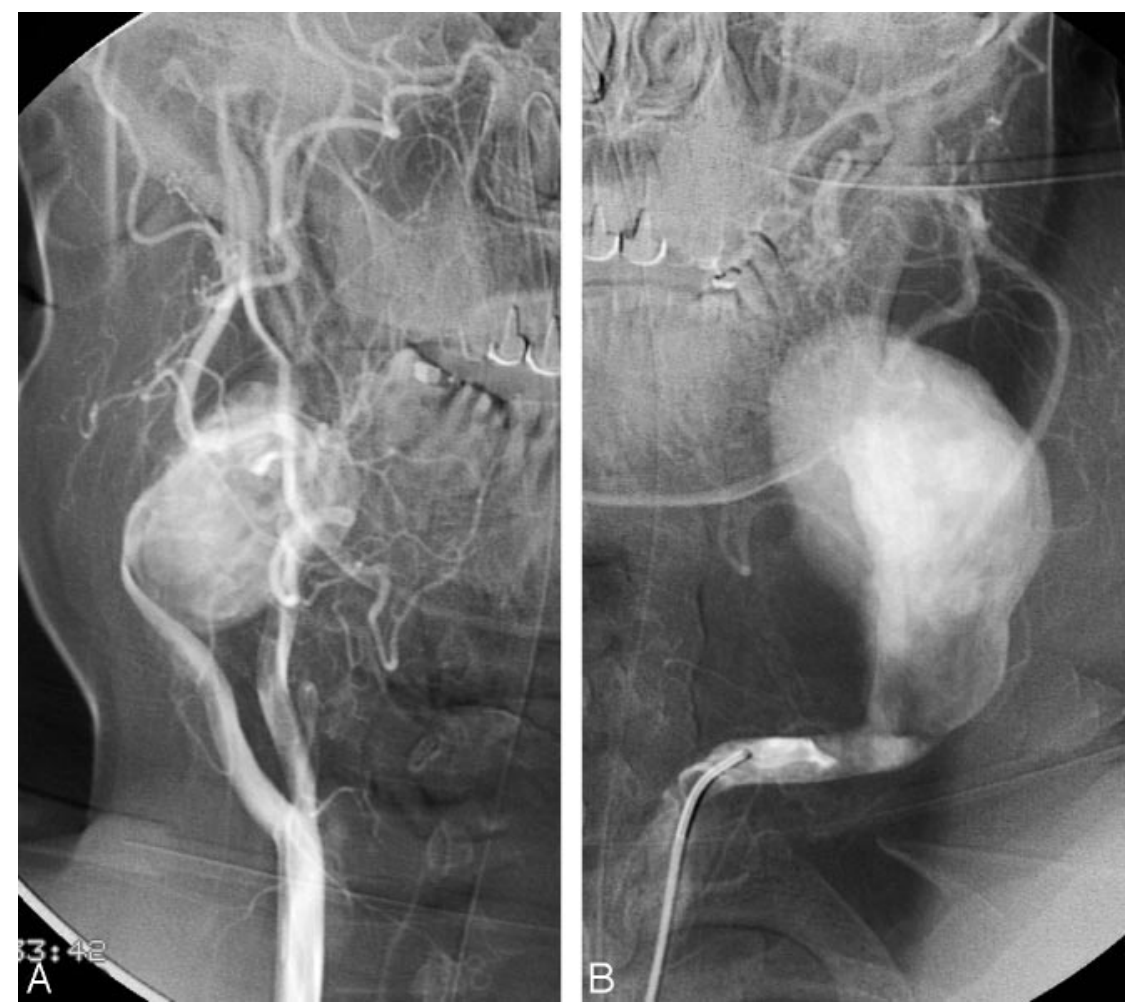

Fig 1. A, Right common carotid artery-injected angiography reveals a right extracranial ICA giant aneurysm. B, Left common carotid artery-injected angiography reveals a left extracranial ICA giant aneurysm.
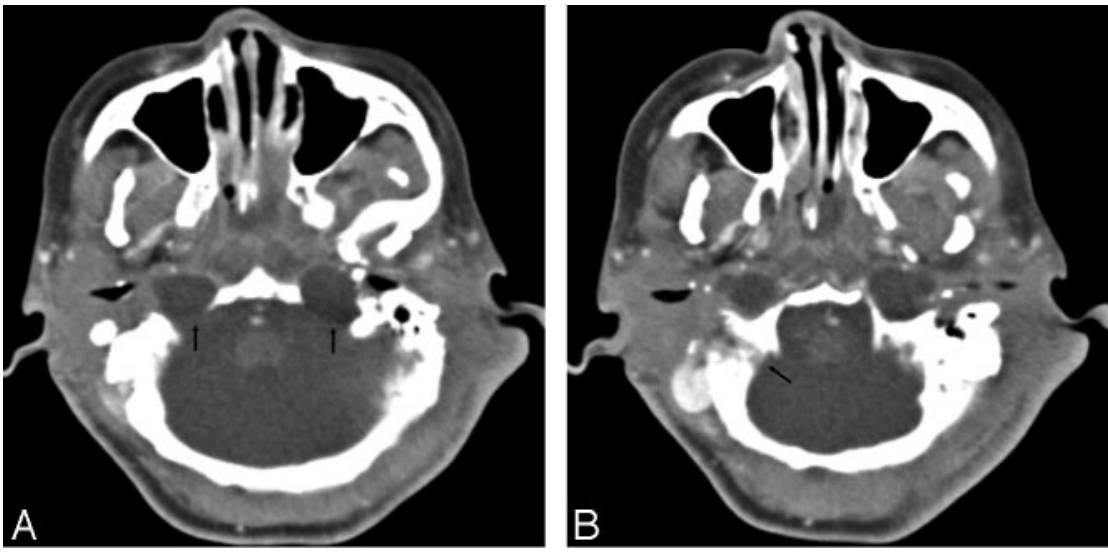

Fig 2. A, Contrast-enhanced axial CT scan reveals focal bony defects bilaterally at the jugular foramen and hypoglossal canal with exophytic meningoceles (arrows). B, Contrast-enhanced axial CT scan reveals a right lambdoid suture defect (arrow). The drainage pathway of the right sigmoid sinus is noted to run aberrantly through the lambdoid sutures because of a space-occupying meningocele at the right jugular foramen.

surrounded by plexiform neurofibromas, suggesting that some vascular wall weakening may have been due to compression and/or infiltration.

However, recent work in genetics and molecular biology strongly suggests that most vascular lesions in patients with NF-1 are due to decreased expression of the gene product neurofibromin. Neurofibromin normally acts as a tumor-suppressor product through downregulation of reticular activating system (RAS)-induced signaling. ${ }^{11,12}$ In normal vascular tissue, neurofibromin is strongly expressed by both endothelial and smooth muscle cells. ${ }^{6,11,12}$ Mice engineered to have increased RAS signaling through a different mutation than the one underlying NF-1 develop neointimal hyperplasia and luminal occlusion similar to what is seen in patients with NF-1 who have cerebrovascular complications. ${ }^{11}$ Work with mouse vascular smooth muscle cells and with cells obtained from patients with NF-1 shows that loss of neurofibromin results in increased proliferation and migration of vascular smooth muscle cells. ${ }^{11}$ Work with mice engineered to have homozygous loss of the homolog NF-1 in smooth muscle cells has produced several findings with significant implications for patients with NF-1. ${ }^{12}$ Under normal conditions, these mice develop and live comparably to their genetically healthy peers. However, they show a markedly pathologic response to vascular injury. There is marked intimal hyperproliferation and increased proliferation of smooth muscle cells. If research confirms that vascular abnormalities in NF-1 are acquired through abnormal responses to injury and are not congenital 

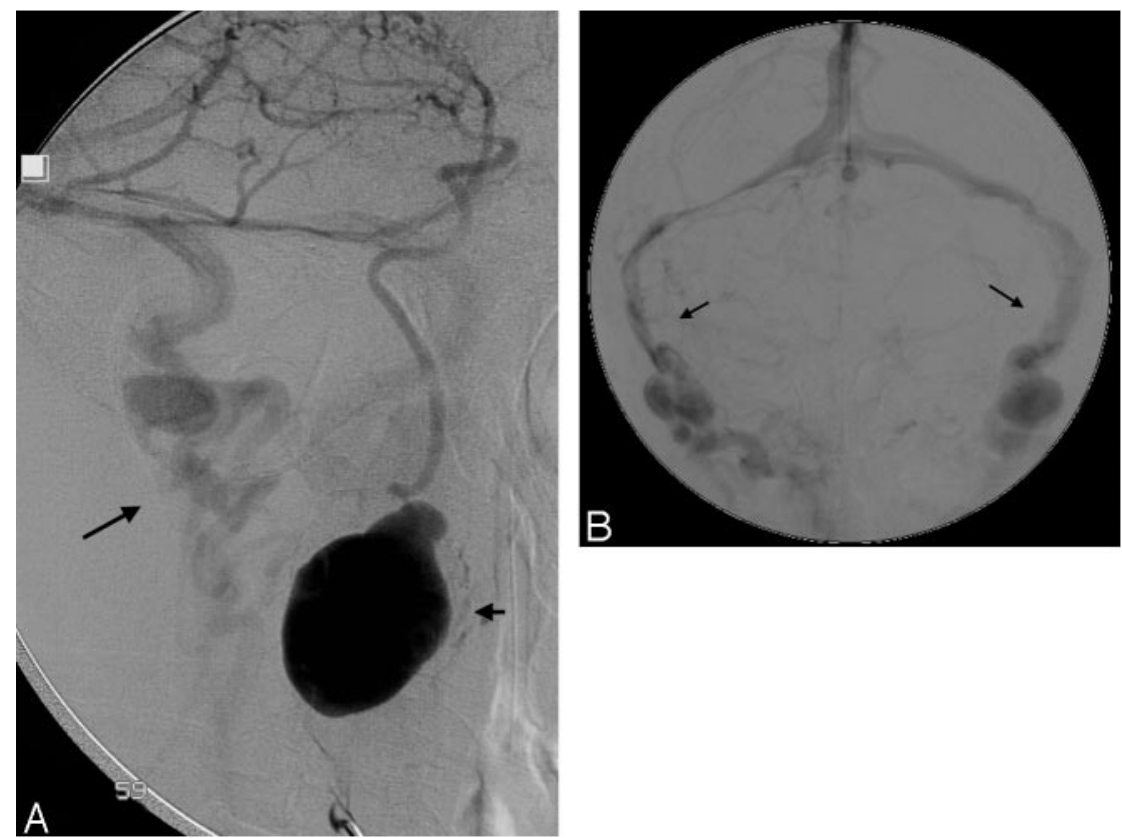

Fig 3. $A$, Lateral view of the right cervical ICA taken with prolonged-injection angiography reveals an aneurysm (short arrow) and right aberrant jugular venous drainage (long arrow) through the lambdoid suture defect at the posterior neck. $B$, Venous phase of right carotid artery-injected angiography reveals bilateral sigmoid sinuses (arrows) running aberrantly through the lambdoid suture defects with aberrant jugular venous drainage.

in nature with progression with time, targeted therapies may become available to prevent or treat the vasculopathy.

On the basis of our experience and review of the literature, we recommend detailed imaging of patients with NF-1 and apparent vasculopathy to clarify vascular structure and blood flow patterns. Depending on the individual patient, the study may include CT or MR imaging or both, as well as digital subtraction or other angiographic techniques. We believe CT angiography is better than MR angiography for identification of active bleeding due to its ability to identify extravasation of contrast. Even with recent technical advancements in MR angiography, resolution of contrast-enhanced MR angiography is still inferior to that of CT angiography. Besides, MR angiography using the time-of-flight technique is often hindered by turbulent flow-caused artifact, especially at the carotid bifurcation site. In addition, we recommend that clinicians have a high degree of suspicion for lesions in bone or other tissues that may be significant in treatment planning. The single review we identified noted that most vascular lesions are asymptomatic at diagnosis. ${ }^{6}$ However, we saw our patient develop sudden-onset symptoms related to her vascular lesions, and a previous case report noted presentation with sudden-onset symptoms. ${ }^{7}$ It would be prudent to assume that NF-1-associated vascular lesions may become symptomatic unexpectedly.

\section{References}

1. Berg BO. Chromosomal abnormalities and neurocutaneous disorders. In Goetz CG, ed. Textbook of Clinical Neurology. 2nd ed. Philadelphia: Saunders Elsevier; 2003

2. Nopajaroonsri C, Lurie AA. Venous aneurysm, arterial dysplasia, and nearfatal hemorrhages in neurofibromatosis type 1. Hum Pathol 1996;27:982-85

3. Malecha MJ, Rubin R. Aneurysms of the carotid arteries associated with von Recklinghausen's neurofibromatosis. Path Res Pract 1992;188:145-47

4. Rosser TL, Vezina G, Packer RJ. Cerebrovascular abnormalities in a population of children with neurofibromatosis type 1. Neurology 2005;64:553-55

5. Rasmussen SA, Yang Q, Friedman JM. Mortality in neurofibromatosis 1: an analysis using U.S. death certificates. Am J Hum Genet 2001;68:1110-18

6. Hamilton SJ, Friedman JM. Insights into the pathogenesis of neurofibromatosis 1 vasculopathy. Clin Genet 2000;58:341-44

7. Smith BL, Munschauer CE, Diamond N, et al. Ruptured internal carotid aneurysm resulting from neurofibromatosis: treatment with intraluminal stent graft. J Vasc Surg 2000;32:824-28

8. Zhao J-Z, Han X-D. Cerebral aneurysm associated with von Recklinghausen's neurofibromatosis: a case report. Surg Neurol 1998;50:592-96

9. Roth TC, Manness WK, Hershey BL, et al. Complex vertebral arteriovenous fistula and ruptured aneurysm in neurofibromatosis: a therapeutically challenging case. Skull Base Surg 2000;10:35-41

10. Tanaka T, Hasegawa Y, Kanki T, et al. Combination of intravascular surgery and surgical operation for occipital subcutaneous arteriovenous fistula in a patient with neurofibromatosis type 1 [in Japanese]. No Shinkei Geka 2002;30:309-13

11. Li F, Munchhof AM, White HA, et al. Neurofibromin is a novel regulator of RAS-induced signals in primary vascular smooth muscle cells. Hum Mol Genet 2006;15:1921-30. Epub 2006 Apr 27.

12. Xu J, Ismat FA, Wang $T$, et al. NF1 regulates a Ras-dependent vascular smooth muscle proliferative injury response. Circulation 2007;116:2148-56. Epub 2007 Oct 22 